\title{
Gli apparati interferometrici per il tracking $(*)$
}

\author{
A. Gexino
}

Ricevuto il 19 Febbraio 1963

Problemi sempre nuovi si pongono nell'esereizio di un Poligono missilistico a causa dell'amento della velocita dei missili, dell'aumento delle gittate, del perfezionamento dei sistemi di guida con conseguente aumento delle precisioni richieste alla strumentazione, della sempre crescente necessita di ottenere i risultati del lancio in tempo reale, delle esigenze dell'addestramento dei reparti e cosi via.

Prestazioni teconicamente sempre piu esigenti si richiedono alla strumentazione per tenersi aggiornata con le necessita; fino ad un certo punto la tecnica si e spinta su due vie parallele ed altrettanto rediditizie; il perfezionamento dei sistemi ottici ed il potenziamento dei pulse-madar.

Specialmente in quest'ultimo campo si e cosi arrivati a radar con prestazioni veramente eccezionali come l'FPS/16 della R.C.A. ed altri ron caratteristiche similari.

Pero il costo sempre cerescente di tali apparati, la necessita di arrivare a direttivita di fascio oltremodo spinte per poter migliomare la precisione del sistema, la sempre minore superficie equivalente dei bersagli, la impossibilità di funzionare in presenza di contromisure, ma, specialnente, la limitazione insita nel sistema di poter seguire nu solo bersaglio alla volta, hamo posto in evidenza che la tecnica del pulse-palar ha pressoche raggiunto i massini limiti consentiti.

D'altro canto i sistemi ottici, con i quali si possono ottenere importanti prestazioni speceialmente sull'assetto del bersaglio e sono insensibili alle contromisure, hanno limitazioni di impiego evidenti se si pensi, per esempio, alle questioni di visibilità, ai problemi di acquisizione e così via.

Da cio l'esigenza dell'impiego di techiche nuove.

(*) Nota presentata al 2o Congresso Intemazionale Tecnico Scientifico dello Spazio. Roma, 19-23 Giugno 1962. 
I radar Doppler (e meglio ancora i pulse-Doppler) sembrerebbero risolvere molti dei nuovi probleni che si pongono, tuttavia essi hamo in se molte limitazioni quali ad esempio una vasta zona di Doppler zero attorno al traverso, un campo operativo di velocita piuttosto limitato per cui e azzardato esprimere un giudizio specialmente a causa della limitata esperienza di impiego sui campi di tiro.

La tecmica della comparazione di fase ha, viceversa, originato una serie di apparecchiature che, con un termine non perfettamente ortodosso, vengono definite "interferometriche" le quali per la semplicita di concezione, il rosto abbastanza ridotto e, specialmente, per le prestazioni ottenibili, la elasticità d'impiego e la insensibilita alle contromisure sembrano, non a torto, in America i mezzi piu irlonei per le necessita delle prove missilistiche.

Le apparecohiature piu conosciute in questo campo sono l'AZUSA della Convair (che puo essere considerato il apostipite dellat serie), il SECOR, il COTAR e derivati della CUBIC, il MISTRAL della General Electric ed altri di minore importanzal.

Non intendo in questa sede fare un confronto tra questi sistemi. Tuttavia mi e necessario fare riferimento ai sistemi CUBIC perche, oltre ad essere quelli piu usati negli U.S.A., essi rappresentano la famiglia piu completa, sono costruiti anche in Italia e sono in corso di adozione nel Poligono di Salto di Quirra, in Sardegna.

I sistemi interferometrici si articolano principalnente su due elementi fondamentali:

a) un apparato misuratore di distanza (D.1E e) rhe utilizza il tempo di transito da un trasmettitore a terra ad un ripetitore a bordo e vicerersa, per determinare lo "slant range".

b) un apparato nisuratore di angoli (AME) che sfrutta la differenza di tempo con eni un segnale emesso da bordo arriva a terra ad una coppia di antenne, opportunamente distanziate, allo scopo di determinare i coseni direttori della linea di sito della provenienza del segnale stesso.

In ambedue i casi, la tecnica della misuma is quella del confronto di fase.

Essa si estrinsea nella ben nota relazione rhe lega ma lunghezza con la frequenza e l'angolo di fase di un fenomeno ondulatorio; relazione che, supposto ronosciuto il valore della velocita di propagazione, si esporime con la formula

$$
D=\frac{\varphi}{2 \pi} \lambda
$$


ove $D$ e una lunghezza, $\lambda$ la lunghezza d'onda del fenomeno ondulatorio e $\varphi$ il suo angolo di fase.

Esaminiamo ora il principio di funzionamento del DME.

Un trasmettitore emette una frequenza portante $f_{1}$, opportunamente modulata da una frequenza $f_{2}$; un apparato di bordo, ricevendo le emissione da terra, ritrasmette la frequenza $f_{2}$, come modulazione di una portante $f_{3}$, ma, e questo e essenziale, ne mantiene invariatal la fase.

In tale maniera, prescindendo dalle portanti, possiamo schematizzare il funzionamento riferendoci alla sola frequenza $f_{2}$.

La distanza $D$ tra la stazione a terra ed il trasponditore e evidentemente

$$
D=\frac{1}{2} \frac{\Phi}{2 \pi} \lambda-\frac{1}{2} \frac{n+\varphi}{2 \pi} \lambda
$$

Mentre $n$ rappresenta il numero delle ambiguita, è evidente che l'accuratezza nella misura di $\varphi$ rappresenta la accuratezza nella misura della distanza $D$.

Immaginiamo di impiegare per $f_{2}$ una frequenza avente una lunghezza d'onda di 2.000 piedi; poiche, anche con fasometri elettromeccanici, non vi e difficolta a leggere il grado (ed il mezzo grado) di fase, e evidente che l'accuratezza nella misura della distanza oscilla tra 1,5 e 3 piedi circar.

Rimangono le ambiguita.

Se contemporaneamente alla frequenza $f_{2}$, usiamo altre frequenze esattamente sottomultiple di quest'ultima, il numero di ambiguita sara, per ogni nuova modulante, $K$ volte inferiore a quelle di $f_{2}$, essendo $K$ il rapporto fra $f_{2}$ e le singole frequenze. Sono elenrati alcuni esempi di conbinazioni di frequenza più commemente usate per eliminare le ambiguità.

Naturalmente laccuratezal sala $K$ volte minore per cui e logico aftermare che alla frequenza maggiore a affidato il compito di effettuare la misura "molto precisa" ", mentre alle altre si chiede solo di eliminare le ambiguita man mano che la distanza cresce, e le misure relative sono da considerarsi, rispettivamente, come " precisa " " medial " grossolana " e " molto grossolana ".

Per determinare la posizione di un punto nello spazio occorrono almeno tre distanze, per cui la combinazione di almeno 3 stazioni DME forma il sistema SECOR, naturalmente in unione con il trasponditore di bordo.

Allo scopo di non complicare gli apparati e opportuno usare per tutte le stazioni DME del sistema le stesse frequenze, sia portanti che 


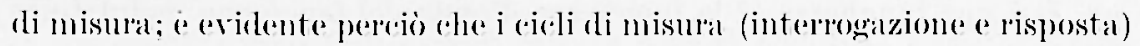
di ogni stazione della catena doverano essere distinti fra lono pere cui il tempo di lavoro di ogni stazione sam limitato, discontinuo e sequenziato.

Una qualsiasi stazione della catema assmme il ruolo di Master e dispone di un codifieatore di tempo in base al quale viene ordinata, via trasponder, la sequenza ceiclica di interrogazione di tutte le stazioni del sistema.

Ogni stazione pero a completamente antonoma per quanto rignarda la misura per cui e pensabile che, per voli fuori clabla portata di un grappo di stazioni, la catena possa essere prolungata passando man mano la funzione di Master.

Se la posizione di tutte le stazioni del sistema e conosciuta con la necessalia precisione rispetto and una terma di assi cartesiani, le coordinate del trasponditore sono esattamente determinabili con uma operazione matematica, che, nella piin semplice espressione consiste nella risoluzione del sistema di tre equazioni della sfera.

E orvio che se rengono impiegate contemporaneamente $N$ stazioni DME, si aramo tanti sistemi quante sono le combinazioni di $N$ equazioni a tre a tre; se ne possono dedurre le conseguenze di preceisione ot tenibili eon il metodo dei valori quadratici medi.

Sempre sul principio del confonto di fase funziona laalto apparato. l'AME.

Il segnale di una sorgente s, distante $R$ dal centro della linea di base $L$ di due antenne segue due pereorsi generalnente diversi tra loro per cui fra le due antemne esiste una differenza di fase proporzionale alla differenza dei pereorsi.

Se osserviano, come is nella realta, che $R$ a molto maggeione di $L$ possiamo considerare $\alpha=90^{\circ}$, ossia, esprimendoci in temini di lumgherzal d'onda e di angolo di fase risulteria

$$
D=a-b=\frac{\theta}{2 \pi} \wedge=K / \cos \theta
$$

ove $K$ e la mistura deela linea di base in lumghezza domela e $p$ e il solito angolo di fase.

Poniamo l'attenzione sugli ultimi due termini della eguaglianza

$$
\varphi-2 \pi K \cos \theta
$$

in cou is definito un rapporto tra la misura della differenza di fase far le antemme ed il cos 0.

E evidente (ehe ogni qualvolta il coseno, nel suo (ampo di valiabilita da 0 a 1 , assume il valore di un sottomultiplo di $K$, il valore di $p$ diventa 
eguale ad un multiplo di $2 \tau$, rioe zero. Abbiamo pereio $2 K$ ambiguita nel campo da 0 a $180^{\circ}$.

$\mathrm{E}$ evidente che nell'intervallo tra due ambiguita, $\varphi$ assume tutti $\mathrm{i}$ valori tra 0 e $2 \pi$; percio se immaginiamo di avere un $K$ di 50 (cioe antenne distanti $50 \lambda$ ) la variazione del coseno che produce un ciclo completo di $\phi$, i di 0,02 .

Se, come visto pere il DMLE, noi riusciano a misurare il grado fed il mezzo grado) di fase, l'acenuatezza nella misura di cos 0 oscilla fra 27,5 e 55 p.p.m.:

Se la linea di base a di 128 , laccuratezza a fra 10 a 20 p.p.m.

Si noti che in pratica questi risultati sono oftenibili con normali fasometri elettromeccenturi.

Dobbiamo eliminare le $2 K$ ambiguita.

Inpiegando sulla stessa linea di base diverse coppie di antenne, con $K$ decrescenti, i evidente che le ambiguita samanno sempre meno fino a sparire per $K=1 / 2$, cioe per una coppla di antenne distanti mezza lunghezza d'onda.

Similmente per quanto fatto per il DME, possiamo considerate " molto precisa "a misuma fatta con la coppia di antenne avente la base magegiore e, via via, "preceisa ", "media " e "grossolana" quella fatta con le coppie di anteme a base ravvicinata, le quali hamo il compito principale di eliminare le ambiguita.

Abbiamo quindi stabilito uno strumento capace di misurare, con estrena precisione, il coseno dell'angolo che la direzione di proventenza dei segnali forma con la linea di base delle antemne.

E noto dalla geometria che il luogo dei punti per i quali i costante la differenza tra le distanze da due punti noti, costituisce un iperboloide di rotazione.

Nel nostro caso per ogni valore di $\varphi$ viene determinato un iperboloide, che possiamo confondere con il cono asintottico, avente il vertice sulla mezzeria della linea di base, l'asse lungo l'allineamento delle antemne e la semiapertura 0 , ormai biunirocamente determinata dalla misura di $\phi$.

La linea di sito cerecatal è ma generatrice del como.

is di fondamentale importanza notare che il cos 0 a il rero coseno direttore della linea di sito rispetto alla linea di base e non, cone ni e "apitato di legegere qualche volta anche in documenti teconici ufficiali, langolo fra la base delle antenne a la proiezione della linea di sito sul piano verticale passante pere la prima.

Una interpretazione del genere oltre ad essere inesatta dal punto di vista matematico a assolutamente errata dal punto di vista fisico, 
per cui, se fosse rera, tutta la filosofia del funzionamento del sistema satrebbe inpossibile.

Due AME, rioe due allineamenti di anteme, perfettamente nguali ed ortogonali far loro, definiscono due coni la cui intersezione e finalmente la linea di sito cerata.

Si noti ancola che l'apparato COTAR (cosi viene chiamata la combinazione di due AME) non fornisce, rome i radar, il valore degli angoli, ma direttamente il valore dei coseni direttori della linea di sito rispetto ad una terna cartesiana di cui gli allineamenti delle antenne sono gli assi $x$ ed $y$ mentre la $z$ e normale al piano delle antenne.

Si può redere come si presenta in realta il piano di antenne COTAR.

La combinazione di 2 COTAR posti a notevole distanza fra loro forma quello che si definisce sistema BICOTAR.

E evidente che la conoscenza esatta della posizione dei due COTAR e la misura di quattro coseni direttori, consentono di ricavare in modo corretto le coordinate del bersaglio con formula che tutti ronoscono.

Un'altá combinazione interessante a quella di un DME e di un COTAR per originare il sistema DME-COTAR. Esso esegue il tracking fornendo, per il calcolo delle coordinate del bersaglio, una distanza e due coseni direttori.

Ci avviciniamo evidentemente al tipo di tracking che si effettua con i radar (2 dati angolari e uno di distanza), ma (e mi preme molto osservarlo) il calcolo numerico e molto pii semplice, infatti non or. corrono le trasformazioni dei dati di uscita degli angoli in funzione trigonometriche perche gli interferometrici forniscono, per la loro natura, direttamente i coseni.

I sistemi che abbiamo esaminato (SECOR-BICOTAR-IDHECOTAR) sono le piu semplici combinazioni possibili tra i due apparati base (DME ed $\mathrm{AME}$ ) e sono idonei per il tracking di un bersaglio, cioe ognuno di essi è paragonabile ad un ladar.

Pero (e qui e uno dei pregi fondamentali di questi apparati rispetto ai radar) i sistemi interferometrici per la loro stessa filosofia di funzionamento e per la loro costruzione, possono facilmente eseguire il trareking di nolti bersagli contemporaneamente senza bisogno di essere multiplati, come nel caso dei radar.

Ritengo necessario, prima di esaminare questi apparati che diremo "multipli ", richiamare l'attenzione sull'importanza di questa flessibilita di impiego.

Prendiamo ad esempio i asisi in cui lo scopo del tracking e proprio quello di determinare la posizione reciprocal di parecehi mobili in volo 
(es.: lancio di missili aria-aria, controllo del traffico aereo attorno ad un aeroposto, sicurezza del poligono, ece.) od anche quando si debbono affrontare problemi di simulazione (es. la distanza di fuga di un aereo rispetto al fronte d'urto di una esplosione atomica o la posizione reciproca di due bersagli pilotati nel caso di addestramento, ecc.).

Innanzitutto e evidente l'economia nel costo dell'installazione; infatti mentre un radar puo seguire un solo bersaglio e per $N$ bersagli occorrono $N$ radar, con l'impiego di apparecchiature interferometriche basta un solo sistema con alcuni componenti raddoppiati.

Se prendiamo poi in considerazione i problemi di acquisizione o, ancora piu importanti, quelli della riarquisizione quando per cause varie il bersaglio viene perduto, l'economia ea ancola piu evidente.

Oggi i radar-tracking sono genemalmente asserviti a sistemi ottici o a radar di ricerca o a sistemi di antenne autopuntanti, comunque e buona regola prevedere una doppia copertura dello spazio interessato, mentre con un solo sistema interferometrico multiplo l'inseguimento e romunque sempre assicurato.

Mediamente per un Poligono avente una gittata dai 60 ai $100 \mathrm{~km}$, quando operino 3 bersagli (intercettore, missile e velivolo bersaglio) sono necessari almeno 10 radar contro un solo sistema MATTS.

Si noti, per inciso, che l'uso dei radar e relativi asservimenti comporta l'impiego di una massa di personale specializzato che e completamente inutile negli apparati interferometrici; e questo mi sembra un altro vantagerio rimarchevole.

Ma vi e ancora una osservazione tecnica, che riguarda la precisione e che sfugge alla prima osservazione superficiale.

Abbiamo premesso che lo scopo principale del tracking è quello di rilevare contemporaneamente le traiettorie di vari bersagli per dedurne il comportamento reciproco, verificandosi determinati eventi.

IIi sembra percio ehe rilevare ogni singola traiettoria con mezzi diversi, una dall'altra, poi confrontarle, avendo come unico parametro coordinatore il tempo, dia, a causa della diversita degli errori propri dei vari mezzi di rilevamento, risultati molto piu imprecisi di quelli che si possono ottenere se il sistema di tracking e unico per tutti i bersagli e gli errori, prevedibili e imprevedibili, sono uguali per tutti.

Inoltre con il rilevamento radarico ad un bersaglio per volta è assolutamente necessario (a meno di non mettere tutti i radar coincidenti) effettuare tutti i calcoli di parallasse prima del confronto delle traiettorie; questi calcoli rengono aboliti con il sistema di rilevamento multiplo, ove le traiettorie sono gia riferite ad un unico sistema di coordinate - 
Trasceuro altri vantaggi, che pure sarebbero da ronsiderane, rome: minore quantita di frequenze in gioco, riduzione di cause di avaria, ecce.

L'idea che i sistemi interferometrice inseguono vali bersagli contemporaneamente a tahmente generalizzata, che oggi negli U.S.A., in Francia, in Srezia ed anche da noi quasi non si prende piin in considerazione il loro impiego come sistemi all un bersaglio.

Per rendere un DME ed un AME capaci di inseguire due o piu bersagli sono impiegate due tereniche diverse: il cosi detto "Frequency Sharing" "ed il cosidetto "Time Sharing"

Con il primo, arlottato necessariamente nei sistemi BICO'TAR, geli emettitori di bordo funzionano con frequenze leggermente diverse fra loro.

E evidente che bastera disporre, per ogni coppia di antenne, di tanti ricevitori e di tanti fasometri quanti sono i bersagli, per avere i coseni rirettori di ognumo di essi, esattamente nello stesso tempo e con gli stessi errori, e percio gia idonei ad essere impiegati per un controllo delle traiettorie e delle posizioni reciproche in rolo.

Si ha pero l'inconveniente che la distanza tra le antenne a tiplo intero di lunghezza d'onda della sola frequenza fondamentale $f_{0}$, cioce di quella emessa da un bersaglio.

Per la corretta valutazione dei coseni direttori di tutti gli altri bersagli oceore un fattore di correzione, per eni

ore

$$
\cos \theta_{o}=\frac{f_{o}}{f_{1}} \cos \theta_{m}=\frac{f_{o}}{f_{o}+\Delta f_{a}+\Delta f_{i}} \cos \theta_{m}
$$

$\cos \theta_{0}$ e il coseno rercato

$\cos 0_{m}$ a il roseno misurato

fo a la frequenza fondamentale del sistema di antenne

$f_{1}$ a la frequenza impiegata

1f. a la rlifferenza conoseiuta tra la frequenza fondamentale e quella impiegata

Afi a la deviazione misurata delle frequenzo dal valore nominale.

Eे superfluo segnalare come nel termine $A f_{i}$ sia compreso, e quindi nisurabile, anche l'eventuale effetto Doppler.

Ia tecnica "Frequency Sharing" consente, per ragioni teconiche, l'estensione del DIIE a due soli bersagli; si preferisce pereio aldottare per il SECOR e per il DAECOTAR la tecnica del "Time Slaaring"

Sfruttando la discontinuita di funzionamento delle stazioni DME e la loro sequenza ricliea si ottiene il funzionamento multiplo imponendo una seconda sequenza per cui i trasponditori di bordo, rispondono aiclicomente a tutte le stazioni di terar che li interogano ciclicamente. 
L evidente che la frequenza di interrogazione a quella di risposta possono rimanere le stesse e non occome multiplarle, pere cui in ogni stazione e sufficiente disporre di un solo ricevitore, conne nei sistemi pere un solo bersaglio, ma oceorreranno tanti fasometri quanti sono i bersangli e l'imput dei fasometri dovra essere comandato dal dispositivo di sequenza.

Nascono cosi i sistemi interferonetrici piin noti: MIDAS, il MATTS il MIOPT.AR, ece. (apace di inseguire fino a $3-4$ e piu bersengli.

Ad Atlantic City is in funzione un MOPTAR per un controllo del traffico aereo per conto del Centro Sperimentale Nazionale delle Apparacechiature di Assistenza al Folo (N.SFEO) della Federal Ariation Ageney (FAN) attrezzato per 5, ma capace di oltre 200 aerei (e si pensal di arrivale a 400$)$.

Il SECOR erd il MOPTAR hamo tuttavia l'inconveniente di esigere a bordo un trasponditore che puo creare difficolta di installazione su veicoli molto piccoli; viceversa il COTAR, il MIDAS ed il MITTS possono inseguire il bersaglio solo che esso disponga a borlo di ma emittente qualsiasi; per questa ragione essi rengono genemalnente costruiti per funzionare nella banda $225 \div 260$ MHz., nom importa se e come modulati, che come es noto e la banda usata per le telemisure.

Il MATTTS, che e stato onologato dalle forze amate Americane con la sigla $\mathrm{A} / \mathrm{GSQ}-29$, non e che la evoluzione piu modelna del MIDAS in quanto quest'ultimo si limita a formie in forma analogica i coseni direttori del rilevamento di doe o tre bersagli e calcolare, in tempo reale ma sempre in forma analogica, i vettori "Miss distance") (cioe distanza tra il missile e il bersaglio) ed "escape distance" (cioc distanzal tá il missile e l'aereo intercettatore).

Nel MATTS le uscite sono state completamente digitalizzate per cui si ragginnge una migliore preceisione. I dati sono immediatamente inseribili in una calcolatrice digitale per tutte le manipolazioni matematiche necessarie per ottenere (sempre che la calcolatrice abbia adeguate possibilità) tutti i paranetri (che si clesidemo (traieftoria, distanze relative, velocita, aceelerazioni, previsione di punto di impatto, controllo della siculezzal, exc(e).

Basta esaminare uno schena a blocehi per sincerarsi che la disponibilita in tempo reale di tutta questa massa di rati, consente di effettuare qualsiasi operazione di traceiamento di traiettorie su appositi tavoli, telepuntamento di cineteodoliti radar e cinetelescopi, comandi e ordini qualsiasi (es. lancio automatico del missile quando il bersaglio a in un certo punto della rotta, ordine ad un satellite di emettere informazioni in rleterminati punti dell'orbita e cosi via). 
L'uso di fasometri digitali, nei qualo lo sfasamento fra l'onda in ingresso ed il segnale di referenza viene misurato in cifra binaria di $2^{10}$, in modo che un ciclo risulta diviso in $102 t$ parti, consente di affermare che non e assolutamente assurdo pretendere da questi apparati le preeisioni di 1 piede, nella misura della distanza e di 10 parti per un milione, nella misura del coseno.

La tecnologia costruttiva di questi apparati non e nulla di trascendentale, ma negli schemi sono evidenti l'impiego abbondante di tecuiche avanzate di feedbak, regolazioni accurate di circuiti critici, precisione nella costruzione mecoanica e aceuratissima scelta delle componenti.

Oggi poi si va sviluppando in modo particolare la tecnica dei ricevitori correlati e coerenti l'uso dei quali permette di sfruttare un'altra singolarita di questi sistemi: la indipendenza teorica della precisione delle grandezze dal valore assoluto delle grandezze stesse; e quindi possibile concepire di utilizzare, senza alcuna modifica o particolari accorgimenti, gli apparati interferometrici in campi di portata che non hanno ronfronti con nessum altro mezzo conosciuto.

Un esperimento pratico effettuato con un aereo a bordo del quale erano state installate due antenne distanti di $4,70 \mathrm{mt}$, ha dato come risultato che il massimo errore compiuto, nella misura dell'intervallo fra le 2 antenne, e stato di $280 \mathrm{~mm}$ mentre il velivolo si trovara a circa $20 \mathrm{~km}$ di distanza.

Pel quanto la sua frequenza fosse tenuta segreta e quindi i filtri necessari preparati solo all'ultimo momento, la capsula spaziale di Glemn e stata rilevata ed inseguita con un sistema MATTS installato a Tyndall $A F B$ in Florida. Si notino gli errori assoluti lungo i 3 assi cartesiani che sono molto piccoli se si considera la piccola base tra le stazioni.

La geometria del rolo e del piazzamento delle stazioni a terra e un fattore determinante per l'arcuratezza dei rilevamenti ron i sistemi interferometrici.

Quando è possibile conoscere a priori l'altezza e la rotta che presumibilmente fara il bersaglio, a abbastanza facile determinare quale deve essere la ubicazione delle stazioni a terra per ottenere la precisione desiderata; tuttavia considerazioni pratiche di accessibilita dei punti scelti o di costo (come nel caso dei campi d'antenna del COTAR) impediscono di avere sempre la dislocazione ideale.

Sono stati effettuati numerosi studi in questo ampo anche per ovviare ad altri inconvenienti come le riflessioni dorute a terreni non piani e per eliminare la degradazione dei risultati ai bassi angoli. 
Per siti ideali, con antemne onni-direzionali, si possono arere errori nella misura della fase di $1^{0}$ o 20 , mentre nelle peggiori condizioni di installazione questo errore di fase sale fino a $10^{\circ}$ e $20^{\circ}$. Questo fa decerescere noterolmente l'aceuratezza fino a 50 p.p.m. con antenne distanziate di 50 lunghezze d'onda. Si e sentita percio la neressita di fare i ampi d'antemne di $256 \%$ per cui anche nelle peggiori condizioni l'aceulatezal rimane compresa nella desiderata misura di 10 p.p.m.

Per i bassi angoli, specialmente sulla riva del mare, hanno dato ottimi risultati l'uso di eampi di antenna inclinati sull'orizzonte per diminuire la degradazione geometrica della precisione.

Per terreni ondulati, come la maggior parte in Italia, si e dimostrata utile l'adozione di antenne direttive, del tipo "fan-beam ", con angolo di apertula verticale abbastanza piccolo per evitare i multi path e angolo azimutale sufficientemente grande per non incorrere in problemi di acquisizione.

In Italia ed altrove, questi sistemi interferometrici hamno aruto ed lamno tuttora molti oppositori. La maggior parte di essi, forse per tradizione, ha voluto contrappore a questa tecnica, nuova ed ancola piena dei problemi che accompagnano tutte le cose nuove, la pii sperimentata e collamblata tecnica radaristica.

Uno degli argomenti piu forti che si e cercato di opporre all'alozione di questi sistemi is stato quello dell'affidabilita.

Dal 23 Ottobre al 3 Novembre del 1961 si sono svolte sulla Base Aerea di Tyndall le gare nazionali di tiro aria-aria tra $\mathrm{i}$ Reparti dell'U.S.A.F. dotati di missile Genio.

Durante questi lanci (e mi affido alle sole cifre Ufficiali) furono effettuate 38 missioni delle quali 31 valide agli effetti della valutazione del tiro; di quelle ammullate ma sola fu per ambiguita nei dati del MIATTS, tutte le altre furono invalidate per altre cause. E per queste prove il MLATS ebbe a funzionare per 155 ore contro le 152 preventivate con ma percentuale operativa del $100 \%$.

Sul poligono della Nary di Point-Arguello in California nel periodo dal 13 Ottobre 1960 al 13 Lugrio 1961 il sistema COTAR, installato per la sicurezza del Poligono, ha partecipato a 138 operazioni, sotto il controllo della Federal Electric Corporation, con una affidabilita del $97,1 \%$.

Concludo con un confronto che ritengo necessario tra l'impiego degli apparati interferometriei e clei ralar-pulse per l'uso di tracking.

Esaniniamo i principali parametri che influiscono sulla scelta dei sistema da impiegale: accuratezza del rilevamento, affidabilita del siste- 
ma, portata e problemi di acquisizione. apparati di bordo, semplicita operativa, schemi di calcolo, costo.

Abbiamo sforato aleme di questi parametri ma vorrei richiamare lantenzione su alcune considerazioni particolari.

Circa l'aceurateza abbiamo visto che gli interferometrici sono notevolmente piu preceisi dei malar; pero, si dice, essi hanno una degladazione per bassi angoli per' cui se ne sconsiglia l'impiego per determinati tracking a bassa quota. Ora, a prescindere dalle precauzioni che si possono prendere per eliminare questo inconveniente, quale radar non ha degradazioni notevoli per angoli al disotto dei $4_{-5}^{\circ}$ ? Se poi si considera che, in pratica, l'accumatezza media nella determinazione della posizione e dellordine di grandezza di 5 piedi per gli interferometrici, di $25 \div 50$ piedli per i radar della classe FPS 16 a di 150 piedi per radar come r.MPQ 12, l'MIPQ 18, l'MSG 1, i lecito affermare che i primi si possono permettere il lusso di ma degradazione 10 volte superione a quella dei ladar, senza intaceare il loro diritto ad essere messi a confronto.

IIo gia fornito alcune infomazioni pratiche sulla afficlabilita dei sistemi, ma rorrei osservare che essi, non avendo, come i radar, organi mereanici in movimento ed essendo concettualmente piu semplici non possono essere di scarsa affirlabilita, naturalmente purche siano costruiti con mal certa accurateza.

Per oftenere alte precisioni nei radar a necessario ridure al minimo le dimensioni trasversali del fascio per cui si arriva a veri e propri pennelli lineiformi tenuti puntati sul bersaglio da servomeceanismi comandati datensioni errore.

Questo pero va a scapito dell'acquisizione iniziale e della riacequisizione nel (aso (abbastanza frequente) di sganciamento del bersaglio per molteplici ragioni. Per evitare questo inconveniente si deve fare forzatamente uso di sistemi ausiliari di puntamento (raclar di sorveglianZa, colomnine ottiche, antenne autopuntanti, ecce) e si deve assiemare la copertuma della zona interessata con almeno due radar tracking.

Nei sistemi interferometrici non i viceversa necessario puntare l'antemara sul bersaglio in quanto essi funzionano solo e proprio perche il bersaglio a in zona ed emette. E pereio evidente che, essendo sistemi "cooperatori" che hamno bisogno di una emissione da borclo, lacequisizione viene ricondotta nei liniti di un problema di propagazione.

Rimane eventualmente la portata.

Essa negli interferometrici is unicamente legata alla potenza del trasmettitore di bordo ed alla sensibilita dei ricevitori; agendo specialmentes su questi ultimi e usando la teconica della portante coerente e della 
comerazione di fase si possono ottenere portate di centinaia di miglia ron minime potenze a bordo.

Communue a evidente dalla filosofia dei sistemi che non vi e questione di portate nel fumzionamento del COTAR; eventualmente ri potra essere una questione di ambiguita nel SECOR, quando la distanza superi la lunghezza d'onda della misura grossolana, ma mi sembra che leerrore sia talmente grande da essere riconoscibile ron il solo buon senso.

Ial portata dei ladar è viceversa limitata dagli asservimenti di distanza e dalle potenze d'eco disponibili; soltanto modifiche sostanziali possono eliminare la prima limitazione mentre potenze gigantesche, anteme ciclopiche per concentrare semple pin il fascio, rinforzatori di eco e trasponditori, sono necessari per aumentale i livelli delleco.

L'uso dei trasponditori a microonde per i radar pone anche dei problemi di "package " a bordo. Infatti essi hamno dimensioni strettamente obbligate dall'uso delle guide d'onda, hamno antenne ingombranti, richiedono un assorbimento di energia abbastanza grande ed hamno pesi sensibili.

Iso stesso problema di "package") si pone anche per i trasponditori del SECOR; tuttavia non essendo legati a problemi mecoanici, essi sono molto flessibili nella forma e adattabili a qualsiasi esigenza di bordo, ed inoltre debbono emettere piccole potenze, anche la disponibilita di encruia puo essere limitata.

I sistemi cOTAR non presentano invere nessuna difficolta perche essi sfruttano qualsiasi emissione da bordo, e, nel caso che non vi sia a borilo una emittente, un trasmettitore $\mathrm{CW}$, miniaturizzato alle dimensioni di un sigaro avana, es sufficiente perche il cotar determini la posizione del bersigglio.

Questa semplicita di funzionamento trova riscontro nella sempliceita operativa dei sistemi interferometrice rispetto ai radar.

Vogliamo per un momento seguire lo svolgimento delle operazioni durante un lancio di missile aria-alria contro un velivolo bersaglio radioguidato?

Le due Stazioni MATTS ricerono il segmale da bordo del velivolo bersaglio, ne rilevano i coseni direttori che', forniti in forma digitale and un calcolatore, consentono automaticamente e senza particolari elaborazioni il rilevamento della traiettoria. Non appena il velivolo intercettore ed il missile sono in vista ottica, dalle stazioni vengono rilevati i loro coseni direttori, forniti dalla stessa calcolatrice, i quali permettono il traceiamento delle traiettorie. 
Dal momento del lancio fino alla fine dell'operazione la calcolatrice fornisce oltre le traiettorie anche la "miss-distance " e la "escape-distance".

Tut to e automatico, non richiede intervento umano, basta solo che vi siano i segnali da rilevare.

Se la stessa operazione deve essere svolta con i radar, supponendo di fare la doppia ropertura, abbiamo bisogno di un radar di ricerca, 6 roppie di puntatori ottici, 6 radar tracking con i relativi operatori, 3 calcolatrici per la trasformazione dei rilevamenti angolari dei 3 mobili in funzione trigonometriche, 3 digitalizzatori di dati e finalmente il calcolatore di traiettoria.

Ogni commento is superfluo.

Vorrei pero sottolineare un dettaglio.

Ise formule per la determinazione delle coordinate del punto partendo dai rilevamenti, comportano, in qualsiasi forma, l'uso di funzioni trigonometriche seno e coseno.

I sistemi interferometrici ci danno, per il loro principio di funzionamento i valori di queste funzioni e si puo procedere nei calcoli senza bisogno di ulteriori manipolazioni.

I dati angolari dei radar escono, per loro natura, sotto forma di angoli e per la trasformazione in funzioni circolari dobbiamo fare uso di resolver da cui non possiamo aspettarei ne una estrema accuratezza ne un'alta discriminazione dei valori di uscita, oppure sono necessari calcolatori digitali con memorie rilevanti per ricordare tutti i valori dei seni e coseni per piccole variazioni di angoli.

E inutile parlare di costi perche il confronto appena fatto sulla mole di strumentazione necessaria, nei due casi, parla da se.

Non eredo quindi azzardato asserire che l'era dei radar-pulse come strumentazione principe sui Poligoni, va lasciando il posto alla nuova tecnica che si va affermando.

Fonti altamente qualificate dell'Esercito, della Marina e dell'Aviazione Americama confermano questa osservazione quando, in documenti Ufficiali, dichiarano che i sistemi interferometrici producono dati piu accurati dei pulse-radar, quantunque alcuni pulse-radar di precisione forniscano dati di accumateza comparabile con i sistemi ottici.

E che l'avvenire della tecnica del tracking interferometrico sia luminoso, lo si puo osservare dal fatto che i responsabili della strumentazione dei Poligoni Americani hanno deriso di non stanziare pii fondi per gli studi dei pulse-radar, ma di sostenere noterolmente i sistemi a comparazione di fase un po' erroneamente detti interferometrici. 